1 Hacettepe Journal of Mathematics and Statistics

h Volume 48 (1) (2019), 200-212

\title{
Two classes of risk model with diffusion and multiple thresholds: the discounted dividends
}

\author{
Wuyuan Jiang*†
}

\begin{abstract}
In this paper, we consider the present value of total dividends until ruin in a perturbed risk model with two independent classes of risks under multiple thresholds, in which both of the two inter-claim times have phase-type distributions. We obtain the integro-differential equations for the moment-generating function and the $r$ th moment of discounted dividend payments. Explicit expressions for the expectation of discounted dividend payments are derived if the two classes claim amount distributions both belong to the rational family.
\end{abstract}

Keywords: Two classes of claims, Diffusion process, Dividend payments, Multiple thresholds, Phase-type distribution.

2010 AMS Classification: 62P05; 91B30

Received : 22.09.2017 Accepted : 28.06.2018 Doi : 10.15672/HJMS.2018.602

\section{Introduction}

The discounted sum of dividend payments until ruin is an important quantity in assessing the quality of a dividend barrier strategy in insurance risk theory, which has been studied in some papers and books, see e.g. [1], [3], [5], [8], [10], [13].

Recently, some researchers consider the ruin measures for a risk model involving two independent classes of risks in the actuarial literature. Among them, [11] considered the expected discounted penalty functions by assuming that the two claim number processes are independent Poisson and generalized Erlang(2) processes. [15] supposed that the claim number processes are independent Poisson and generalized Erlang(n) processes, respectively, in which the Laplace transforms of the expected discounted penalty functions are obtained. As an extension to these papers, [7] investigated the same ruin measures in the risk model with two classes of renewal risk processes by assuming that both of the two claim number processes have phase-type inter-claim times.

*Department of Mathematics, Hunan Institute of Science and Technology, Yueyang 414006, China, Email: csujw@163.com

$\dagger$ Corresponding Author. 
There is a particular attention in considering risk models with multi-threshold dividend strategies. For instance, [12] discussed the Gerber-Shiu expected discounted penalty function in the compound Poisson risk model with multiple thresholds. [14] extended the corresponding results to a Sparre Andersen model with generalized Erlang $(n)$-distributed inter-claim times. In insurance risk models with multiple thresholds, the premium rate is a step function of the insurer's surplus. The premium policy is effective when the insurer intend to keep a fixed retention ratio on its revenues and pays bonuses as an incentive to its policyholders.

[9] investigated the discounted penalty function for two classes of risk processes with diffusion and multiple thresholds, where both of the two claim number processes have phase-type inter-claim times. It is natural to ask for the results on the discounted sum of dividend payments until ruin for a corresponding risk model. The rest of the paper is structured as follows. Section 2 describes the risk model. In Section 3, we derive systems of integro-differential equations for the moment generating function. In Section 4, integrodifferential equations for the moments of discounted dividend payments are obtained. Section 5 presents the main results and derives explicit expressions for the expectation of discounted dividend payments when two classes claim amount distributions both belong to the rational family. Section 6 gives a numerical example.

\section{Notation and model description}

The surplus process $R(t)$ perturbed by diffusion satisfies

$$
R(t)=u+c t-S(t)+\sigma B(t), \quad t \geq 0,
$$

where $u \geq 0$ is the initial surplus, $c$ denotes the insurer's premium income per unit time, $\{B(t) ; t \geq 0\}$ is a standard Brownian motion and $\sigma>0$ is the dispersion parameter, and the aggregate-claim process $\{S(t): t \geq 0\}$ is defined by

$$
S(t)=\sum_{i=1}^{N_{1}(t)} X_{i}+\sum_{i=1}^{N_{2}(t)} Y_{i}, \quad t \geq 0
$$

where $\left\{X_{1}, X_{2}, \cdots\right\}$ and $\left\{Y_{1}, Y_{2}, \cdots\right\}$ are independent and identically distributed (i.i.d.) positive random variables representing the successive individual claim amounts from the first and the second class, respectively. The random variables $\left\{X_{1}, X_{2}, \cdots\right\}$ are assumed to have common cumulative distribution function $F(x)=1-\bar{F}(x), x \geq 0$, with probability density function $f(x)=F^{\prime}(x)$, of which the Laplace transform is $\tilde{f}(s)=\int_{0}^{\infty} e^{-s x} f(x) d x, s \in \mathbb{C}, \mathbb{C}$ denotes the complex space. Similarly, common cumulative distribution function, density function and the Laplace transform of the density function of $\left\{Y_{1}, Y_{2}, \cdots\right\}$ are given by $G(x)=1-\bar{G}(x), x \geq 0, g(x)=G^{\prime}(x)$ and $\tilde{g}(s)=\int_{0}^{\infty} e^{-s x} g(x) d x$. The renewal processes $\left\{N_{1}(t) ; t \geq 0\right\}$ and $\left\{N_{2}(t) ; t \geq 0\right\}$ denote the number of claims up to time $t$ caused by the first and the second class of claim respectively, and are defined as follows.

$$
\begin{aligned}
& N_{1}(t)=\sup \left\{n: T_{1}+T_{2}+\cdots+T_{n} \leq t\right\}, \\
& N_{2}(t)=\sup \left\{n: V_{1}+V_{2}+\cdots+V_{n} \leq t\right\},
\end{aligned}
$$

where the i.i.d. interclaim times $\left\{T_{1}, T_{2}, \cdots\right\}$ have common cumulative distribution function $K_{1}(t), t \geq 0$ and density function $k_{1}(x)=K_{1}^{\prime}(x)$, and $\left\{V_{1}, V_{2}, \cdots\right\}$ have common cumulative distribution function $K_{2}(t), t \geq 0$ and density function $k_{2}(x)=K_{2}^{\prime}(x)$.

In addition, we suppose that $\left\{X_{1}, X_{2}, \cdots\right\},\left\{Y_{1}, Y_{2}, \cdots\right\},\left\{N_{1}(t) ; t \geq 0\right\},\left\{N_{2}(t) ; t \geq 0\right\}$ and $\{B(t) ; t \geq 0\}$ are mutually independent, and $c>E\left(X_{1}\right) / E\left(T_{1}\right)+E\left(Y_{1}\right) / E\left(V_{1}\right)$, providing a positive safety loading factor. 
Under the multi-threshold risk model, there are $L$ thresholds $0=d_{0}<d_{1}<\cdots<$ $d_{L-1}<d_{L}=\infty$ such that when the surplus is between the thresholds $d_{l-1}$ and $d_{l}$, dividends are paid continuously at a constant rate $\eta_{l} \geq 0$. Furthermore, we assume $\eta_{1}=0$, namely, when the surplus is below the level $d_{1}$, no dividends are paid, and $\eta_{l}>0$ for $l=2,3, \cdots, L$. Correspondingly, let $c_{l}$ denote the premium rate when $d_{l-1} \leq u<d_{l}$, thus, the net premium rate after dividend payments is $c_{l+1}=c_{1}-\eta_{l+1} \geq 0$. Thus the surplus process $\{R(t) ; t \geq 0\}$ can be expressed as

$$
d R(t)=c_{l} d t+\sigma d B(t)-d S(t), \quad d_{l-1} \leq R(t)<d_{l} .
$$

The time of (ultimate) ruin is defined as $T=\inf \{t \mid R(t) \leq 0\}$, where $T=\infty$ if $R(t)>0$ for all $t \geq 0$. The probability of ruin is $\psi(u)=\operatorname{Pr}(T<\infty)$.

Denote by $D(t)$ the cumulative amount of dividends paid out up to time $t$ and $\delta>0$ the force of interest, then $\mathbb{D}=\int_{0}^{T} e^{-\delta t} d D(t)$ is the present value of all dividends until ruin time $T$. In the following text, we turn to the moment generating function under multiple thresholds,

$$
M(u, y)=E\left[e^{y \mathbb{D}} \mid R(0)=u\right]
$$

(for those values of $y$ where it exists) and the $r$ th moment

$$
W(u, r)=E\left[\mathbb{D}^{r} \mid R(0)=u\right], \quad r \in \mathbb{N} .
$$

Note that $W(u, 0) \equiv 1$. We will always assume that $M(u, y)$ and $W(u, r)$ are sufficiently smooth functions in $u$ and $y$, respectively.

Throughout the text of the paper, all bold-faced letters represent either vectors or matrices and all vectors are column vectors. We assume that the distribution $K_{1}(t)$ of the inter-claim time random variable $T_{1}$ is phase-type with representation $\left(\boldsymbol{\alpha}^{\top}, \mathbf{A}, \mathbf{a}\right)$, where $\boldsymbol{\alpha}^{\top}=\left(\alpha_{1}, \alpha_{2}, \cdots, \alpha_{n}\right)$, with $\alpha_{i} \geq 0, \sum_{i=1}^{n} \alpha_{i}=1, \mathbf{A}=\left(a_{i j}\right)_{i, j=1}^{n}$ is an $n \times n$ matrix with $a_{i i}<0, a_{i j} \geq 0$, for $i \neq j, \sum_{j=1}^{n} a_{i j} \leq 0$, for any $i=1,2, \cdots, n$, and $\mathbf{a}=\left(a_{1}, a_{2}, \cdots, a_{n}\right)^{\top}$ with $\mathbf{a}=-\mathbf{A} \mathbf{e}_{n}$, where $\mathbf{x}^{\top}$ denotes the transpose of $\mathbf{x}$ and $\mathbf{e}_{n}$ denotes a column vector of length $n$ with all elements being one. Following [2], we have $K_{1}(t)=1-\boldsymbol{\alpha}^{\top} e^{\mathbf{A} t} \mathbf{e}_{n}, \quad k_{1}(t)=\boldsymbol{\alpha}^{\top} e^{\mathbf{A} t} \mathbf{a}, \quad t \geq 0$, and

$$
\tilde{k}_{1}(s)=\int_{0}^{\infty} e^{-s t} k_{1}(t) d t=\boldsymbol{\alpha}^{\top}(s \mathbf{I}-\mathbf{A})^{-1} \mathbf{a} .
$$

By the definition of phase-type distributions, each of the inter-claim times $T_{i}, i=1,2, \cdots$, corresponds to the time to absorption in a terminating continuous-time Markov Chain, say, $I_{t}^{(i)}$ with $n$ transient states $\left\{E_{1}, E_{2}, \cdots, E_{n}\right\}$ and one absorbing state $E_{0}$.

The distribution $K_{2}(t)$ of the inter-claim time random variable $V_{1}$ is phase-type with representation $\left(\boldsymbol{\beta}^{\top}, \mathbf{B}, \mathbf{b}\right)$, where $\boldsymbol{\beta}^{\top}=\left(\beta_{1}, \beta_{2}, \cdots, \beta_{m}\right), \mathbf{B}=\left(b_{i j}\right)_{i, j=1}^{m}$ is an $m \times m$ matrix, $\mathbf{b}=\left(b_{1}, b_{2}, \cdots, b_{m}\right)^{\top}$ with $\mathbf{b}=-\mathbf{B e}_{m}$. Then we have $K_{2}(t)=1-\boldsymbol{\beta}^{\top} e^{\mathbf{B} t} \mathbf{e}_{m}$, $k_{2}(t)=\boldsymbol{\beta}^{\top} e^{\mathbf{B} t} \mathbf{b}, t \geq 0$, and $\tilde{k}_{2}(s)=\int_{0}^{\infty} e^{-s t} k_{2}(t) d t=\boldsymbol{\beta}^{\top}(s \mathbf{I}-\mathbf{B})^{-1} \mathbf{b} . J_{t}^{(i)}$ denotes the terminating continuous-time Markov Chain of $V_{i}, i=1,2, \cdots$, with $m$ transient states $\left\{F_{1}, F_{2}, \cdots, F_{m}\right\}$ and one absorbing state $F_{0}$.

Now, we construct a two-dimensional Markov process $\{(I(t), J(t)) ; t \geq 0\}$ by piecing the $\left\{I_{t}^{(i)} ; i=1,2, \cdots\right\}$ and $\left\{J_{t}^{(i)} ; i=1,2, \cdots\right\}$ together,

$$
\begin{gathered}
I(t)=\left\{I_{t}^{(1)}\right\}, 0 \leq t<T_{1}, \quad I(t)=\left\{I_{t-T_{1}}^{(2)}\right\}, T_{1} \leq t<T_{1}+T_{2}, \cdots, \\
J(t)=\left\{J_{t}^{(1)}\right\}, 0 \leq t<V_{1}, \quad J(t)=\left\{J_{t-V_{1}}^{(2)}\right\}, V_{1} \leq t<V_{1}+V_{2}, \cdots .
\end{gathered}
$$

So $\{(I(t), J(t)) ; t \geq 0\}$ is the underlying state process with states $\left\{\left(E_{1}, F_{1}\right),\left(E_{2}, F_{1}\right), \cdots\right.$, $\left.\left(E_{n}, F_{1}\right),\left(E_{1}, F_{2}\right),\left(E_{2}, F_{2}\right), \cdots,\left(E_{n}, F_{2}\right), \cdots,\left(E_{1}, F_{m}\right),\left(E_{2}, F_{m}\right), \cdots,\left(E_{n}, F_{m}\right)\right\}$, initial distribution $\boldsymbol{\gamma}=\boldsymbol{\beta} \otimes \boldsymbol{\alpha}$, where $\otimes$ denotes the Kronecker product of two matrices. 
For $k=1,2 ; i=1,2, \cdots, n ; j=1,2, \cdots, m$, let $M^{(k)}(u, y)$ denote the moment generating function of $\mathbb{D}$ if the ruin is caused by a claim from class $k$ and $R(0)=u . M_{i j}^{(k)}(u, y)$ denotes the moment generating function of $\mathbb{D}$ when the ruin is caused by a claim from class $k$ and initial state $\left(I_{0}^{(1)}, J_{0}^{(1)}\right)=\left(E_{i}, F_{j}\right)$, then the moment generating function can be written as

$$
M^{(k)}(u, y)=\gamma^{\top} \mathbf{M}^{(k)}(u, y)
$$

where $\mathbf{M}^{(k)}(u, y) \equiv\left(M_{11}^{(k)}(u, y), M_{21}^{(k)}(u, y), \cdots, M_{n 1}^{(k)}(u, y), M_{12}^{(k)}(u, y), M_{22}^{(k)}(u, y), \cdots\right.$, $\left.M_{n 2}^{(k)}(u, y), \cdots, M_{1 m}^{(k)}(u, y), M_{2 m}^{(k)}(u, y), \cdots, M_{n m}^{(k)}(u, y)\right)^{\top}$. Thus

$$
M(u, y)=\gamma^{\top} \mathbf{M}(u, y)=\boldsymbol{\gamma}^{\top}\left[\mathbf{M}^{(1)}(u, y)+\mathbf{M}^{(2)}(u, y)\right]
$$

$W_{i j}(u, r)$ denotes the $r t h$ moment of $\mathbb{D}$ if $\left(I_{0}^{(1)}, J_{0}^{(1)}\right)=\left(E_{i}, F_{j}\right)$, then the moment can be computed by

$$
W(u, r)=\gamma^{\top} \mathbf{W}(u, r)
$$

where $\mathbf{W}(u, r) \equiv\left(W_{11}(u, r), W_{21}(u, r), \cdots, W_{n 1}(u, r), W_{12}(u, r), W_{22}(u, r), \cdots, W_{n 2}(u, r)\right.$, $\left.\cdots, W_{1 m}(u, r), W_{2 m}(u, r), \cdots, W_{n m}(u, r)\right)^{\top}$.

\section{The moment generating function}

Let $\frac{\partial}{\partial u}$ and $\frac{\partial}{\partial y}$ denote the differentiation operators with respect to (w.r.t.) $u$ and $y$, respectively.

3.1. Theorem. The vectors $\mathbf{M}^{(k)}(u, y), d_{l-1} \leq u<d_{l}, l=1,2, \ldots, L, k=1,2$ satisfy the following partial integro-differential system, respectively,

$$
\begin{aligned}
& \left(\frac{\sigma^{2}}{2} \frac{\partial^{2}}{\partial u^{2}}+c_{l} \frac{\partial}{\partial u}-y \delta \frac{\partial}{\partial y}+y \eta_{l}\right) \mathbf{M}^{(1)}(u, y)+I_{m \times m} \otimes \mathbf{A} \mathbf{M}^{(1)}(u, y)+ \\
& \mathbf{B} \otimes I_{n \times n} \mathbf{M}^{(1)}(u, y)+I_{m \times m} \otimes\left(\mathbf{a} \boldsymbol{\alpha}^{\top}\right) \int_{0}^{u} \mathbf{M}^{(1)}(u-x, y) f(x) d x+ \\
& \left(\mathbf{b} \boldsymbol{\beta}^{\top}\right) \otimes I_{n \times n} \int_{0}^{u} \mathbf{M}^{(1)}(u-x, y) g(x) d x+\left(\mathbf{e}_{m} \otimes \mathbf{a}\right) \bar{F}(u)=\mathbf{0},
\end{aligned}
$$

and

$$
\begin{aligned}
& \left(\frac{\sigma^{2}}{2} \frac{\partial^{2}}{\partial u^{2}}+c_{l} \frac{\partial}{\partial u}-y \delta \frac{\partial}{\partial y}+y \eta_{l}\right) \mathbf{M}^{(2)}(u, y)+I_{m \times m} \otimes \mathbf{A M}^{(2)}(u, y)+ \\
& \mathbf{B} \otimes I_{n \times n} \mathbf{M}^{(2)}(u, y)+I_{m \times m} \otimes\left(\mathbf{a} \boldsymbol{\alpha}^{\top}\right) \int_{0}^{u} \mathbf{M}^{(2)}(u-x, y) f(x) d x+ \\
& \left(\mathbf{b} \boldsymbol{\beta}^{\top}\right) \otimes I_{n \times n} \int_{0}^{u} \mathbf{M}^{(2)}(u-x, y) g(x) d x+\left(\mathbf{b} \otimes \mathbf{e}_{n}\right) \bar{G}(u)=\mathbf{0}
\end{aligned}
$$

where $I_{n \times n}$ denotes the $n \times n$ identity matrix, $\mathbf{0}$ denotes a column vector of length $m n$ with all elements being 0. $\bar{F}(u)=\int_{u}^{\infty} f(x) d x$ and $\bar{G}(u)=\int_{u}^{\infty} g(x) d x$.

Proof. Taking into account an infinitesimal time interval $(0, d t)$ for $d_{l-1} \leq u<d_{l}, l=$ $1,2, \ldots, L$, there are four possible events regarding to the occurrence of the claim and change of the environment: (1) no claim arrival and no change of state; (2) a claim arrival but no change of state; (3) a change of state but no claim arrival; (4) two or more events 
occur. Using the total expectation formula, yields

$$
\begin{aligned}
& M_{i j}^{(1)}(u, y) \\
& =e^{y \eta_{l} d t}\left\{\left(1+a_{i i} d t\right)\left(1+b_{j j} d t\right) E\left[M_{i j}^{(1)}\left(u+c_{l} d t+\sigma B(d t), y e^{-\delta d t}\right)\right]\right. \\
& +\left(1+b_{j j} d t\right) \sum_{k=1, k \neq i}^{n}\left(a_{i k} d t\right) E\left[M_{k j}^{(1)}\left(u+c_{l} d t+\sigma B(d t), y e^{-\delta d t}\right)\right] \\
& +\left(1+a_{i i} d t\right) \sum_{h=1, h \neq j}^{m}\left(b_{j h} d t\right) E\left[M_{i h}^{(1)}\left(u+c_{l} d t+\sigma B(d t), y e^{-\delta d t}\right)\right] \\
& +\left(1+b_{j j} d t\right)\left(a_{i} d t\right) \\
& \times E\left[\sum_{s=1}^{n} \alpha_{s} \int_{0}^{u+c_{l} d t+\sigma B(d t)} M_{s j}^{(1)}\left(u+c_{l} d t+\sigma B(d t)-x, y e^{-\delta d t}\right) f(x) d x\right. \\
& \left.+\int_{u+c_{l} d t+\sigma B(d t)}^{\infty} f(x) d x\right]+\left(1+a_{i i} d t\right)\left(b_{j} d t\right) \\
& \left.\times E\left[\sum_{r=1}^{m} \beta_{r} \int_{0}^{u+c_{l} d t+\sigma B(d t)} M_{i r}^{(1)}\left(u+c_{l} d t+\sigma B(d t)-x, y e^{-\delta d t}\right) g(x) d x\right]\right\} \\
& +o(d t) .
\end{aligned}
$$

By the aid of Taylor expansion, we have

$$
\begin{aligned}
& E\left[M_{i j}^{(1)}\left(u+c_{l} d t+\sigma B(d t), y e^{-\delta d t}\right)\right] \\
=\quad & M_{i j}^{(1)}(u, y)+c_{l} d t \frac{\partial M_{i j}^{(1)}(u, y)}{\partial u}+y\left(e^{-\delta d t}-1\right) \frac{\partial M_{i j}^{(1)}(u, y)}{\partial y} \\
+ & \frac{\sigma^{2}}{2} d t \frac{\partial^{2} M_{i j}^{(1)}(u, y)}{\partial u^{2}}+o(d t) .
\end{aligned}
$$

Substituting (3.4) into (3.3), after some careful calculations, it follows that

$$
\begin{aligned}
& \left(\frac{\sigma^{2}}{2} \frac{\partial^{2}}{\partial u^{2}}+c_{l} \frac{\partial}{\partial u}-y \delta \frac{\partial}{\partial y}+y \eta_{l}\right) M_{i j}^{(1)}(u, y)+\sum_{k=1}^{n} a_{i k} M_{k j}^{(1)}(u, y)+ \\
& \sum_{h=1}^{m} b_{j h} M_{i h}^{(1)}(u, y)+a_{i}\left(\sum_{s=1}^{n} \alpha_{s} \int_{0}^{u} M_{s j}^{(1)}(u-x, y) f(x) d x+\int_{u}^{\infty} f(x) d x\right) \\
& +b_{j} \sum_{r=1}^{m} \beta_{r} \int_{0}^{u} M_{i r}^{(1)}(u-x, y) g(x) d x=0 .
\end{aligned}
$$

Rewriting (3.5) in matrix form, we conclude (3.1). By similar arguments, we can obtain (3.2).

\section{The moments of discounted dividend payments}

4.1. Theorem. The vector $\mathbf{W}(u, r), d_{l-1} \leq u<d_{l}, l=1,2, \ldots, L$, satisfies the following integro-differential system,

$$
\begin{aligned}
& \left(\frac{\sigma^{2}}{2} \frac{d^{2}}{d u^{2}}+c_{l} \frac{d}{d u}-r \delta\right) \mathbf{W}(u, r)+r \eta_{l} \mathbf{W}(u, r-1)+I_{m \times m} \otimes \mathbf{A W}(u, r)+ \\
& \mathbf{B} \otimes I_{n \times n} \mathbf{W}(u, r)+I_{m \times m} \otimes\left(\mathbf{a} \boldsymbol{\alpha}^{\top}\right) \int_{0}^{u} \mathbf{W}(u-x, r) f(x) d x+ \\
& \left(\mathbf{b} \boldsymbol{\beta}^{\top}\right) \otimes I_{n \times n} \int_{0}^{u} \mathbf{W}(u-x, r) g(x) d x=\mathbf{0},
\end{aligned}
$$

with boundary conditions

$$
\left.\mathbf{W}(u, r)\right|_{u=0}=\mathbf{0},\left.\quad \mathbf{W}(u, r)\right|_{u=d_{l}^{-}}=\left.\mathbf{W}(u, r)\right|_{u=d_{l}^{+}},
$$

and

$$
\left.\frac{d \mathbf{W}(u, r)}{d u}\right|_{u=d_{l}^{-}}=\left.\frac{d \mathbf{W}(u, r)}{d u}\right|_{u=d_{l}^{+}} .
$$


Proof. Adding (3.1) to (3.2) and noting that $\mathbf{M}(u, y)=\mathbf{M}^{(1)}(u, y)+\mathbf{M}^{(2)}(u, y)$, which results in

$$
\begin{aligned}
& \left(\frac{\sigma^{2}}{2} \frac{\partial^{2}}{\partial u^{2}}+c_{l} \frac{\partial}{\partial u}-y \delta \frac{\partial}{\partial y}+y \eta_{l}\right) \mathbf{M}(u, y)+I_{m \times m} \otimes \mathbf{A M}(u, y)+ \\
& \mathbf{B} \otimes I_{n \times n} \mathbf{M}(u, y)+I_{m \times m} \otimes\left(\mathbf{a} \boldsymbol{\alpha}^{\top}\right) \int_{0}^{u} \mathbf{M}(u-x, y) f(x) d x+ \\
& \left(\mathbf{b} \boldsymbol{\beta}^{\top}\right) \otimes I_{n \times n} \int_{0}^{u} \mathbf{M}(u-x, y) g(x) d x+\left(\mathbf{e}_{m} \otimes \mathbf{a}\right) \bar{F}(u) \\
& +\left(\mathbf{b} \otimes \mathbf{e}_{n}\right) \bar{G}(u)=\mathbf{0} .
\end{aligned}
$$

Since $W(u, r)=E\left[\mathbb{D}^{r} \mid R(0)=u\right]$, we have the following representation

$$
\mathbf{M}(u, y)=\mathbf{e}_{m n}+\sum_{r=1}^{\infty} \frac{y^{r}}{r !} \mathbf{W}(u, r) .
$$

Substituting (4.5) into (4.4) and equating the coefficients of $y^{r}(r \in \mathbb{N})$. Then by virtue of $\mathbf{a}=-\mathbf{A} \mathbf{e}_{n}$ and $\mathbf{b}=-\mathbf{B} \mathbf{e}_{m}$. Further, $I_{m \times m} \otimes \mathbf{A} \mathbf{e}_{m n}=-I_{m \times m} \otimes\left(\mathbf{a} \boldsymbol{\alpha}^{\top}\right) \mathbf{e}_{m n}=-\mathbf{e}_{m} \otimes \mathbf{a}$ and $\mathbf{B} \otimes I_{n \times n} \mathbf{e}_{m n}=-\left(\mathbf{b} \boldsymbol{\beta}^{\top}\right) \otimes I_{n \times n} \mathbf{e}_{m n}=-\mathbf{b} \otimes \mathbf{e}_{n}$. Hence, we achieve (4.1).

When $u=0$, ruin is immediate and no dividends are paid. That is to say $\left.\mathbf{W}(u, r)\right|_{u=0}=$ 0. Utilizing the continuity of $\mathbf{W}(u, r)$ and thanks to [16], we have the boundary conditions (4.2) and (4.3).

4.2. Remark. We assume that $m=1$ and $G(0)=1$, from Eq.(4.1), which yields

$$
\begin{aligned}
& \left(\frac{\sigma^{2}}{2} \frac{d^{2}}{d u^{2}}+c_{l} \frac{d}{d u}-r \delta\right) \mathbf{W}(u, r)+r \eta_{l} \mathbf{W}(u, r-1) \\
+\quad & \mathbf{A W}(u, r)+\left(\mathbf{a} \alpha^{\top}\right) \int_{0}^{u} \mathbf{W}(u-x, r) f(x) d x=\mathbf{0},
\end{aligned}
$$

where $d_{l-1} \leq u<d_{l}, l=1,2, \ldots, L$. Furthermore, when $L=2$ and the distribution $K_{1}(t)$ is a generalized $\operatorname{Erlang}(n)$ distribution, we recover Theorem 4.1 in [5] from (4.6), which consider the perturbed renewal risk model with a threshold dividend strategy.

\section{The expectation of discounted dividend payments}

In what follows, we consider the case $r=1$ for $\mathbf{W}(u, r)$, the expectation of discounted dividend payments. For notational convenience, let $\mathbf{W}(u) \equiv \mathbf{W}(u, 1)$. From Theorem 4.1 , we have for $d_{l-1} \leq u<d_{l}, l=1,2, \ldots, L$, that

$$
\begin{aligned}
& \left(\frac{\sigma^{2}}{2} \frac{d^{2}}{d u^{2}}+c_{l} \frac{d}{d u}-\delta\right) \mathbf{W}(u)+\eta_{l} \mathbf{e}_{m n}+I_{m \times m} \otimes \mathbf{A W}(u)+ \\
& \mathbf{B} \otimes I_{n \times n} \mathbf{W}(u)+I_{m \times m} \otimes\left(\mathbf{a} \boldsymbol{\alpha}^{\top}\right) \int_{0}^{u} \mathbf{W}(u-x) f(x) d x+ \\
& \left(\mathbf{b} \boldsymbol{\beta}^{\top}\right) \otimes I_{n \times n} \int_{0}^{u} \mathbf{W}(u-x) g(x) d x=\mathbf{0},
\end{aligned}
$$

and with boundary conditions $\left.\mathbf{W}(u)\right|_{u=0}=\mathbf{0},\left.\mathbf{W}(u)\right|_{u=d_{l}^{-}}=\left.\mathbf{W}(u)\right|_{u=d_{l}^{+}},\left.\frac{d \mathbf{W}(u)}{d u}\right|_{u=d_{l}^{-}}$ $=\left.\frac{d \mathbf{W}(u)}{d u}\right|_{u=d_{l}^{+}}$.

5.1. Laplace transforms. Motivating by [12], we relax the constraint $d_{l-1} \leq u<d_{l}$ in (5.1) and consider the case of $u \geq d_{l-1}$. Let $\mathbf{W}_{l}(u), u \geq d_{l-1}, l=1, \cdots, L$ be the solutions of the following non-homogeneous integro-differential equations:

$$
\begin{aligned}
& \left(\frac{\sigma^{2}}{2} \frac{d^{2}}{d u^{2}}+c_{l} \frac{d}{d u}-\delta\right) \mathbf{W}_{l}(u)+I_{m \times m} \otimes \mathbf{A W}_{l}(u)+\mathbf{B} \otimes I_{n \times n} \mathbf{W}_{l}(u)+ \\
& I_{m \times m} \otimes\left(\mathbf{a} \boldsymbol{\alpha}^{\top}\right)\left[\int_{0}^{u-d_{l-1}} \mathbf{W}_{l}(u-x) f(x) d x+\int_{u-d_{l-1}}^{u} \mathbf{W}(u-x) f(x) d x\right] \\
& +\left(\mathbf{b} \boldsymbol{\beta}^{\top}\right) \otimes I_{n \times n}\left[\int_{0}^{u-d_{l-1}} \mathbf{W}_{l}(u-x) g(x) d x+\int_{u-d_{l-1}}^{u} \mathbf{W}(u-x) g(x) d x\right] \\
& +\eta_{l} \mathbf{e}_{m n}=\mathbf{0}, \quad u \geq d_{l-1} .
\end{aligned}
$$


From the theory of differential equations, it follows that

$$
\mathbf{W}(u)=\mathbf{W}_{l}(u)+\sum_{j=1}^{m n} k_{l j} \Theta_{l j}(u), \quad d_{l-1} \leq u<d_{l},
$$

where $k_{l j}$ is constant coefficient for each $l$ and $j$, and $\boldsymbol{\Theta}_{l j}(u), j=1,2, \cdots, m n$, are $m n$ linearly independent solutions to the associated homogeneous integro-differential equations

$$
\begin{aligned}
& \left(\frac{\sigma^{2}}{2} \frac{d^{2}}{d u^{2}}+c_{l} \frac{d}{d u}-\delta\right) \boldsymbol{\Theta}_{l}(u)+I_{m \times m} \otimes \mathbf{A} \boldsymbol{\Theta}_{l}(u)+ \\
& \mathbf{B} \otimes I_{n \times n} \boldsymbol{\Theta}_{l}(u)+I_{m \times m} \otimes\left(\mathbf{a} \boldsymbol{\alpha}^{\top}\right) \int_{0}^{u-d_{l-1}} \boldsymbol{\Theta}_{l}(u-x) f(x) d x+ \\
& \left(\mathbf{b} \boldsymbol{\beta}^{\top}\right) \otimes I_{n \times n} \int_{0}^{u-d_{l-1}} \boldsymbol{\Theta}_{l}(u-x) g(x) d x=\mathbf{0}, \quad u \geq d_{l-1} .
\end{aligned}
$$

5.1. Remark. When $u \rightarrow \infty$, ruin does not happen all the time and dividends are always paid at a constant rate $\eta_{L}$. So we have $\lim _{u \rightarrow \infty} \mathbf{W}(u)=\frac{\eta_{L}}{\delta} \mathbf{e}_{m n}$. We can found that $\frac{\eta_{L}}{\delta} \mathbf{e}_{m n}$ are really particular solutions of (5.2). It follows from the general theory of differential equations that

$$
\mathbf{W}(u)=\frac{\eta_{L}}{\delta} \mathbf{e}_{m n}+\sum_{j=1}^{m n} k_{L j} \boldsymbol{\Theta}_{L j}(u), \quad u \geq d_{L-1},
$$

Taking a change of variables $z=u-d_{l-1}$ and $\boldsymbol{\Phi}_{l}(z) \equiv \mathbf{W}_{l}(u)=\mathbf{W}_{l}\left(z+d_{l-1}\right)$, then we obtain from (5.2),

$$
\begin{aligned}
& \left(\frac{\sigma^{2}}{2} \frac{d^{2}}{d z^{2}}+c_{l} \frac{d}{d z}-\delta\right) \boldsymbol{\Phi}_{l}(z)+I_{m \times m} \otimes \mathbf{A} \boldsymbol{\Phi}_{l}(z)+ \\
& \mathbf{B} \otimes I_{n \times n} \boldsymbol{\Phi}_{l}(z)+I_{m \times m} \otimes\left(\mathbf{a} \boldsymbol{\alpha}^{\top}\right) \int_{0}^{z} \boldsymbol{\Phi}_{l}(z-x) f(x) d x+ \\
& \left(\mathbf{b} \boldsymbol{\beta}^{\top}\right) \otimes I_{n \times n} \int_{0}^{z} \boldsymbol{\Phi}_{l}(z-x) g(x) d x+\boldsymbol{\Gamma}_{l}(z)=\mathbf{0}, \quad z \geq 0,
\end{aligned}
$$

where

$$
\begin{aligned}
\boldsymbol{\Gamma}_{l}(z)= & I_{m \times m} \otimes\left(\mathbf{a} \boldsymbol{\alpha}^{\top}\right) \int_{0}^{d_{l-1}} \mathbf{W}(x) f\left(z+d_{l-1}-x\right) d x \\
& +\left(\mathbf{b} \boldsymbol{\beta}^{\top}\right) \otimes I_{n \times n} \int_{0}^{d_{l-1}} \mathbf{W}(x) g\left(z+d_{l-1}-x\right) d x+\eta_{l} \mathbf{e}_{m n} .
\end{aligned}
$$

Next define the following Laplace transforms: $\tilde{\boldsymbol{\Phi}}_{l}(s)=\int_{0}^{\infty} e^{-s x} \boldsymbol{\Phi}_{l}(x) d x, \tilde{\boldsymbol{\Gamma}}_{l}(s)=$ $\int_{0}^{\infty} e^{-s x} \boldsymbol{\Gamma}_{l}(x) d x$. Taking Laplace transforms on both sides of (5.6) and rearranging, we have

$$
\begin{aligned}
& {\left[\left(\frac{\sigma^{2}}{2} s^{2}+c_{l} s-\delta\right) I_{m n \times m n}+I_{m \times m} \otimes \mathbf{A}+\mathbf{B} \otimes I_{n \times n}+\right.} \\
& \left.I_{m \times m} \otimes\left(\mathbf{a} \boldsymbol{\alpha}^{\top}\right) \tilde{f}(s)+\left(\mathbf{b} \boldsymbol{\beta}^{\top}\right) \otimes I_{n \times n} \tilde{g}(s)\right] \tilde{\boldsymbol{\Phi}}_{l}(s)= \\
& \frac{\sigma^{2}}{2} \boldsymbol{\Phi}_{l}^{\prime}(0)+p_{l}(s) \boldsymbol{\Phi}_{l}(0)-\tilde{\boldsymbol{\Gamma}}_{l}(s),
\end{aligned}
$$

where $p_{l}(s)=\frac{\sigma^{2}}{2} s+c_{l}, \mathbf{\Phi}_{l}(0)=\mathbf{W}_{l}\left(d_{l-1}\right), \boldsymbol{\Phi}_{l}^{\prime}(0)=\mathbf{W}_{l}^{\prime}\left(d_{l-1}\right)$.

Let $\mathbf{L}_{l}(s)=\left(\frac{\sigma^{2}}{2} s^{2}+c_{l} s-\delta\right) I_{m n \times m n}+I_{m \times m} \otimes \mathbf{A}+\mathbf{B} \otimes I_{n \times n}+I_{m \times m} \otimes\left(\mathbf{a} \boldsymbol{\alpha}^{\top}\right) \tilde{f}(s)+$ $\left(\mathbf{b} \boldsymbol{\beta}^{\top}\right) \otimes I_{n \times n} \tilde{g}(s)$, and $\mathbf{L}_{l}{ }^{*}(s)$ is the adjoint of matrix $\mathbf{L}_{l}(s)$ for $l=1,2, \cdots, L$. Thus, when $\operatorname{det}\left[\mathbf{L}_{l}(s)\right] \neq 0$, we get from $(5.8)$

$$
\tilde{\mathbf{\Phi}}_{l}(s)=\frac{\mathbf{L}_{l}{ }^{*}(s)}{\operatorname{det}\left[\mathbf{L}_{l}(s)\right]}\left(\frac{\sigma^{2}}{2} \boldsymbol{\Phi}_{l}^{\prime}(0)+p_{l}(s) \boldsymbol{\Phi}_{l}(0)-\tilde{\boldsymbol{\Gamma}}_{l}(s)\right) .
$$

For a given $l$ the generalized Lundberg's equations $\operatorname{det}\left[\mathbf{L}_{l}(s)\right]=0$ has exactly $m n$ roots in the right half of the complex plane when $\delta>0$, see e.g. [7] for details. We denote them by $\rho_{l 1}, \rho_{l 2}, \cdots, \rho_{l, m n}$ respectively, and for simplicity, we assume that they are different from each other. 
Divided difference plays an important role in the present paper. Now we recall divided differences of a matrix $\mathbf{L}(s)$ w.r.t. distinct numbers $r_{1}, r_{2}, \cdots$, which are defined recursively as follows:

$$
\mathbf{L}\left[r_{1}, s\right]=\frac{\mathbf{L}(s)-\mathbf{L}\left(r_{1}\right)}{s-r_{1}}, \quad \mathbf{L}\left[r_{1}, r_{2}, s\right]=\frac{\mathbf{L}\left[r_{1}, s\right]-\mathbf{L}\left[r_{1}, r_{2}\right]}{s-r_{2}},
$$

and so on.

Since each element of $\tilde{\mathbf{\Phi}}_{l}(s)$ is finite for all $\Re(s)>0, \rho_{l 1}, \rho_{l 2}, \cdots, \rho_{l, m n}$ are also roots of numerator in (5.9). Utilizing a similar technique to Theorem 4.2 in [7], we obtain from (5.9) the following theorem.

5.2. Theorem. The Laplace transforms of $\boldsymbol{\Phi}_{l}(y)$ for $l=1,2, \cdots, L$ are represented by

$$
\begin{aligned}
& \tilde{\boldsymbol{\Phi}}_{l}(s)= \\
& \frac{\prod_{j=1}^{m n}\left(s-\rho_{l j}\right)}{\operatorname{det}\left[\mathbf{L}_{l}(s)\right]}\left\{\mathbf{L}_{l}{ }^{*}\left[\rho_{l 1}, \cdots, \rho_{l, m n}, s\right]\left(\frac{\sigma^{2}}{2} \boldsymbol{\Phi}_{l}^{\prime}(0)+p_{l}(s) \boldsymbol{\Phi}_{l}(0)-\tilde{\boldsymbol{\Gamma}}_{l}(s)\right)+\right. \\
& \left.\mathbf{L}_{l}{ }^{*}\left[\rho_{l 1}, \cdots, \rho_{l, m n}\right] \frac{\sigma^{2}}{2} \boldsymbol{\Phi}_{l}(0)-\sum_{i=1}^{m n} \mathbf{L}_{l}{ }^{*}\left[\rho_{l 1}, \cdots, \rho_{l i}\right] \tilde{\boldsymbol{\Gamma}}_{l}\left[\rho_{l i}, \cdots, \rho_{l, m n}, s\right]\right\} .
\end{aligned}
$$

5.2. The homogeneous integro-differential equations. The solutions to the associated homogeneous integro-differential equations (5.4) are uniquely determined by the initial conditions $\boldsymbol{\Theta}_{l}\left(d_{l-1}\right)$ and $\boldsymbol{\Theta}_{l}^{\prime}\left(d_{l-1}\right)$. In the following, we apply Laplace transforms to find the solutions of $(5.4)$.

let $z=u-d_{l-1}$ and $\boldsymbol{\Xi}_{l}(z) \equiv \boldsymbol{\Theta}_{l}(u)=\boldsymbol{\Theta}_{l}\left(z+d_{l-1}\right), l=1,2, \cdots, L$, then (5.4) can be rewritten as

$$
\begin{aligned}
& \left(\frac{\sigma^{2}}{2} \frac{d^{2}}{d z^{2}}+c_{l} \frac{d}{d z}-\delta\right) \boldsymbol{\Xi}_{l}(z)+I_{m \times m} \otimes \mathbf{A} \boldsymbol{\Xi}_{l}(z)+ \\
& \mathbf{B} \otimes I_{n \times n} \boldsymbol{\Xi}_{l}(z)+I_{m \times m} \otimes\left(\mathbf{a} \boldsymbol{\alpha}^{\top}\right) \int_{0}^{z} \boldsymbol{\Xi}_{l}(z-x) f(x) d x+ \\
& \left(\mathbf{b} \boldsymbol{\beta}^{\top}\right) \otimes I_{n \times n} \int_{0}^{z} \boldsymbol{\Xi}_{l}(z-x) g(x) d x=\mathbf{0}, \quad z \geq 0 .
\end{aligned}
$$

Taking Laplace transforms on both sides of (5.11) yields

$$
\begin{aligned}
& {\left[\left(\frac{\sigma^{2}}{2} s^{2}+c_{l} s-\delta\right) I_{m n \times m n}+I_{m \times m} \otimes \mathbf{A}+\mathbf{B} \otimes I_{n \times n}+\right.} \\
& \left.I_{m \times m} \otimes\left(\mathbf{a} \boldsymbol{\alpha}^{\top}\right) \tilde{f}(s)+\left(\mathbf{b} \boldsymbol{\beta}^{\top}\right) \otimes I_{n \times n} \tilde{g}(s)\right] \tilde{\boldsymbol{\Xi}}_{l}(s)=\frac{\sigma^{2}}{2} \boldsymbol{\Xi}_{l}^{\prime}(0)+p_{l}(s) \boldsymbol{\Xi}_{l}(0),
\end{aligned}
$$

where $\tilde{\boldsymbol{\Xi}}_{l}(s)=\int_{0}^{\infty} e^{-s x} \boldsymbol{\Xi}_{l}(x) d x$. Then, we have

$$
\tilde{\boldsymbol{\Xi}}_{l}(s)=\frac{\mathbf{L}_{l}^{*}(s)}{\operatorname{det}\left[\mathbf{L}_{l}(s)\right]}\left(\frac{\sigma^{2}}{2} \mathbf{\Xi}_{l}^{\prime}(0)+p_{l}(s) \boldsymbol{\Xi}_{l}(0)\right) \text {. }
$$

Since $\boldsymbol{\Theta}_{l}\left(d_{l-1}\right)=\boldsymbol{\Xi}_{l}(0), \boldsymbol{\Theta}_{l}^{\prime}\left(d_{l-1}\right)=\boldsymbol{\Xi}_{l}^{\prime}(0)$, invert (5.13) leads to

$$
\boldsymbol{\Theta}_{l}(u)=\mathcal{L}^{-1}\left\{\frac{\mathbf{L}_{l}^{*}(s)}{\operatorname{det}\left[\mathbf{L}_{l}(s)\right]}\left(\frac{\sigma^{2}}{2} \boldsymbol{\Theta}_{l}^{\prime}\left(d_{l-1}\right)+p_{l}(s) \boldsymbol{\Theta}_{l}\left(d_{l-1}\right)\right)\right\}, \quad u \geq d_{l-1} .
$$

5.3. Claim sizes with rational Laplace transform. Let us now restrict the further analysis to the case of the claim amount distributions $F(x)$ and $G(x)$ both with rational Laplace transforms, that is,

$$
\tilde{f}(s)=\frac{\mathfrak{q}_{m_{1}-1}(s)}{\mathfrak{q}_{m_{1}}(s)}, \quad \tilde{g}(s)=\frac{\mathfrak{r}_{m_{2}-1}(s)}{\mathfrak{r}_{m_{2}}(s)}, \quad m_{1}, m_{2} \in \mathbb{N}^{+},
$$

where $\mathfrak{q}_{m_{1}-1}(s), \mathfrak{r}_{m_{2}-1}(s)$ are polynomials of degree $m_{1}-1$ and $m_{2}-1$ or less, respectively, while $\mathfrak{q}_{m_{1}}(s)$ and $\mathfrak{r}_{m_{2}}(s)$ are polynomials of degree $m_{1}$ and $m_{2}$ with only negative roots, and satisfy $\mathfrak{q}_{m_{1}-1}(0)=\mathfrak{q}_{m_{1}}(0), \mathfrak{r}_{m_{2}-1}(0)=\mathfrak{r}_{m_{2}}(0)$. Without loss of generality, we assume that $\mathfrak{q}_{m_{1}}(s)$ and $\mathfrak{r}_{m_{2}}(s)$ have leading coefficient 1 . This wide class of distributions includes the Erlang, Coxian and phase-type distributions, and also the mixtures of these. 
Multiplying both numerator and denominator of (5.13) by $\mathfrak{h}(s)$, where $\mathfrak{h}(s)=\left[\mathfrak{q}_{m_{1}}(s) \mathfrak{r}_{m_{2}}(s)\right]^{m n}$. We get for $l=1,2, \cdots, L$ that

$$
\tilde{\boldsymbol{\Xi}}_{l}(s)=\frac{\mathbf{L}_{l}^{*}(s)}{\mathfrak{h}(s) \operatorname{det}\left[\mathbf{L}_{l}(s)\right]}\left(\mathfrak{h}(s) \frac{\sigma^{2}}{2} \boldsymbol{\Xi}_{l}^{\prime}(0)+\mathfrak{h}(s) p_{l}(s) \boldsymbol{\Xi}_{l}(0)\right) \text {. }
$$

It is obvious that the factor $\mathfrak{h}(s) \operatorname{det}\left[\mathbf{L}_{l}(s)\right]$ of the denominator is a polynomial of degree $m n\left(m_{1}+m_{2}+2\right)$ with leading coefficient $\left(\sigma^{2} / 2\right)^{m n}$. Therefore, the equation $\mathfrak{h}(s) \operatorname{det}\left[\mathbf{L}_{l}(s)\right]=0$ has $m n\left(m_{1}+m_{2}+2\right)$ roots on the complex plane. We can factorize $\mathfrak{h}(s) \operatorname{det}\left[\mathbf{L}_{l}(s)\right]$ as follows

$$
\mathfrak{h}(s) \operatorname{det}\left[\mathbf{L}_{l}(s)\right]=\left(\frac{\sigma^{2}}{2}\right)^{m n} \prod_{j=1}^{m n}\left(s-\rho_{l j}\right) \prod_{j=1}^{m n\left(m_{1}+m_{2}+1\right)}\left(s+R_{l j}\right)
$$

where $R_{l j}$ for each $l$ and $j$ has positive real part and we assume that all of them are distinct from each other.

Since $p_{l}(s)$ with degree 1 , the numerator $\mathbf{L}_{l}{ }^{*}(s)\left(\mathfrak{h}(s) \frac{\sigma^{2}}{2} \boldsymbol{\Xi}_{l}^{\prime}(0)+\mathfrak{h}(s) p_{l}(s) \boldsymbol{\Xi}_{l}(0)\right)$ in (5.15) is a polynomial with degree less than $m n\left(m_{1}+m_{2}+2\right)$ for each $l$. By the partial fraction decomposition, we get

$$
\tilde{\boldsymbol{\Xi}}_{l}(s)=\sum_{j=1}^{m n} \frac{\boldsymbol{\vartheta}_{l j}}{s-\rho_{l j}}+\sum_{j=1}^{m n\left(m_{1}+m_{2}+1\right)} \frac{\boldsymbol{\chi}_{l j}}{s+R_{l j}}, \quad s \in \mathbb{C},
$$

where $\boldsymbol{\vartheta}_{l j}$, for $j=1,2, \cdots, m n$, and $\chi_{l j}$, for $j=1,2, \cdots, m n\left(m_{1}+m_{2}+1\right)$, are the coefficient matrices defined respectively by

$$
\boldsymbol{\vartheta}_{l j}=-\frac{\mathbf{L}_{l}^{*}\left(\rho_{l j}\right)\left(\mathfrak{h}\left(\rho_{l j}\right) \frac{\sigma^{2}}{2} \boldsymbol{\Xi}_{l}^{\prime}(0)+\mathfrak{h}\left(\rho_{l j}\right) p_{l}\left(\rho_{l j}\right) \boldsymbol{\Xi}_{l}(0)\right)}{\left(\frac{\sigma^{2}}{2}\right)^{m n}\left[\prod_{k=1}^{m n\left(m_{1}+m_{2}+1\right)}\left(R_{l k}+\rho_{l j}\right)\right]\left[\prod_{i=1, i \neq j}^{m n}\left(\rho_{l i}-\rho_{l j}\right)\right]},
$$

and

$$
\chi_{l j}=\frac{\mathbf{L}_{l}{ }^{*}\left(-R_{l j}\right)\left(\mathfrak{h}\left(-R_{l j}\right) \frac{\sigma^{2}}{2} \mathbf{\Xi}_{l}^{\prime}(0)+\mathfrak{h}\left(-R_{l j}\right) p_{l}\left(-R_{l j}\right) \boldsymbol{\Xi}_{l}(0)\right)}{\left(\frac{\sigma^{2}}{2}\right)^{m n}\left[\prod_{k=1}^{m n}\left(\rho_{l k}+R_{l j}\right)\right]\left[\prod_{i=1, i \neq j}^{m n\left(m_{1}+m_{2}+1\right)}\left(R_{l i}-R_{l j}\right)\right]}
$$

Inverting (5.17) yields

$$
\boldsymbol{\Xi}_{l}(z)=\sum_{j=1}^{m n} \boldsymbol{\vartheta}_{l j} e^{\rho_{l j} z}+\sum_{j=1}^{m n\left(m_{1}+m_{2}+1\right)} \chi_{l j} e^{-R_{l j} z}, \quad z \geq 0 .
$$

To conclude, we have

5.3. Theorem. If the claim-size distributions $F(x)$ and $G(x)$ both belong to the rational family, then the solutions of the associated homogeneous integro-differential equations (5.4) are given by

$$
\boldsymbol{\Theta}_{l}(u)=\sum_{j=1}^{m n} \boldsymbol{\vartheta}_{l j} e^{\rho_{l j}\left(u-d_{l-1}\right)}+\sum_{j=1}^{m n\left(m_{1}+m_{2}+1\right)} \chi_{l j} e^{-R_{l j}\left(u-d_{l-1}\right)},
$$

(5.21) $u \geq d_{l-1}, l=1,2, \cdots, L$,

where $\boldsymbol{\vartheta}_{l j}$ and $\chi_{l j}$ are given by (5.18) and (5.19), respectively. 
Next, we turn to derive the expressions of $\mathbf{W}_{l}(u)$, for $l=1,2, \cdots, L$. For this purpose, multiplying both numerator and denominator of (5.10) by $\mathfrak{h}(s)$, by virtue of $(5.16)$ and then canceling the same factor $\prod_{j=1}^{m n}\left(s-\rho_{l j}\right)$, we derive from $(5.10)$ that

$$
\begin{aligned}
& \tilde{\boldsymbol{\Phi}}_{l}(s)=\frac{1}{\left(\frac{\sigma^{2}}{2}\right)^{m n} \prod_{j=1}^{m n\left(m+m_{2}+\right)}\left(s+R_{l j}\right)} \times \\
& \left\{\mathfrak{h}(s) \mathbf{L}_{l}{ }^{*}\left[\rho_{l 1}, \cdots, \rho_{l, m n}, s\right]\left(\frac{\sigma^{2}}{2} \boldsymbol{\Phi}_{l}^{\prime}(0)-\tilde{\boldsymbol{\Gamma}}_{l}(s)\right)+\right. \\
& \mathfrak{h}(s) \mathbf{L}_{l}{ }^{*}\left[\rho_{l 1}, \cdots, \rho_{l, m n}, s\right] p_{l}(s) \boldsymbol{\Phi}_{l}(0)+\mathfrak{h}(s) \mathbf{L}_{l}{ }^{*}\left[\rho_{l 1}, \cdots, \rho_{l, m n}\right] \frac{\sigma^{2}}{2} \boldsymbol{\Phi}_{l}(0) \\
& \left.-h(s) \sum_{i=1}^{m n} \mathbf{L}_{l}{ }^{*}\left[\rho_{l 1}, \cdots, \rho_{l i}\right] \tilde{\boldsymbol{\Gamma}}_{l}\left[\rho_{l i}, \cdots, \rho_{l, m n}, s\right]\right\}
\end{aligned}
$$

Thanks to [9], which can be rewritten as

$$
\begin{aligned}
& \tilde{\boldsymbol{\Phi}}_{l}(s)=\frac{1}{\left(\frac{\sigma^{2}}{2}\right)^{m n}} \sum_{j=1}^{m n\left(m_{1}+m_{2}+1\right)} \frac{1}{s+R_{l j}}\left\{\mathbf{Q}_{l j}\left(\frac{\sigma^{2}}{2} \boldsymbol{\Phi}_{l}^{\prime}(0)-\tilde{\boldsymbol{\Gamma}}_{l}(s)\right)+\mathbf{H}_{l j} \boldsymbol{\Phi}_{l}(0)\right. \\
& \left.+D_{l j}\left(\mathbf{L}_{l}{ }^{*}\left[\rho_{l 1}, \cdots, \rho_{l, m n}\right] \frac{\sigma^{2}}{2} \boldsymbol{\Phi}_{l}(0)-\sum_{i=1}^{m n} \mathbf{L}_{l}{ }^{*}\left[\rho_{l 1}, \cdots, \rho_{l i}\right] \tilde{\boldsymbol{\Gamma}}_{l}\left[\rho_{l i}, \cdots, \rho_{l, m n}, s\right]\right)\right\} .
\end{aligned}
$$

where $\mathbf{Q}_{i j}, D_{i j}$ and $\mathbf{H}_{i j}$ are given respectively by

$$
\mathbf{Q}_{l j}=\frac{\mathfrak{h}\left(-R_{l j}\right) \mathbf{L}_{l}{ }^{*}\left[\rho_{l 1}, \cdots, \rho_{l, m n},-R_{l j}\right]}{m n\left(m_{1}+m_{2}+1\right)}, \quad D_{l j}=\frac{\mathfrak{h}\left(-R_{l j}\right)}{\prod_{i=1, i \neq j}^{m n\left(m_{1}+m_{2}+1\right)}\left(R_{l i}-R_{l j}\right)},
$$

and

$$
\mathbf{H}_{l j}=\frac{\mathfrak{h}\left(-R_{l j}\right) \mathbf{L}_{l}^{*}\left[\rho_{l 1}, \cdots, \rho_{l, m n},-R_{l j}\right] p_{l}\left(-R_{l j}\right)}{m n\left(\prod_{i=1, i \neq j}^{\left.m_{1}+m_{2}+1\right)}\left(R_{l i}-R_{l j}\right)\right.}
$$

In order to obtain the Laplace inverses of (5.23), we recall the operator $T_{r}$ for a realvalued integrable function $f(x)$ defined by $T_{r} f(x)=\int_{x}^{\infty} e^{-r(u-x)} f(u) d u, r \in \mathbb{C}, x \geq 0$. For properties of the operator $T_{r}$, see [4]. Now, we extend the definition of operator $T_{r}$ for a real-valued integrable function to a matrix function w.r.t. a complex number $r$. If each element is a real-valued integrable function of $x$ in matrix $\boldsymbol{\Psi}(x)$, we define $T_{r} \boldsymbol{\Psi}(x)=\int_{x}^{\infty} e^{-r(u-x)} \boldsymbol{\Psi}(u) d u, r \in \mathbb{C}, x \geq 0$, and it is easy to see that

$$
T_{r_{1}} T_{r_{2}} \boldsymbol{\Psi}(x)=T_{r_{2}} T_{r_{1}} \boldsymbol{\Psi}(x)=\frac{T_{r_{1}} \boldsymbol{\Psi}(x)-T_{r_{2}} \boldsymbol{\Psi}(x)}{r_{1}-r_{2}}, r_{1} \neq r_{2} \in \mathbb{C}, x \geq 0 .
$$

Furthermore, from [6], we can get the Laplace inverse of $\tilde{\boldsymbol{\Psi}}\left[r_{1}, r_{2}, \cdots, r_{n}, s\right]$ as follows

$$
\mathcal{L}^{-1}\left(\tilde{\boldsymbol{\Psi}}\left[r_{1}, r_{2}, \cdots, r_{n}, s\right]\right)=(-1)^{n}\left(\prod_{i=1}^{n} T_{r_{i}}\right) \boldsymbol{\Psi}(x) .
$$

Using (5.26) and inverting (5.23), which results in

$$
\begin{aligned}
\boldsymbol{\Phi}_{l}(z) & =\frac{1}{\left(\frac{\sigma^{2}}{2}\right)^{m n}} \sum_{j=1}^{m n\left(m_{1}+m_{2}+1\right)}\left\{\left(\frac{\sigma^{2}}{2} \mathbf{Q}_{l j} \boldsymbol{\Phi}_{l}^{\prime}(0)+\mathbf{H}_{l j} \boldsymbol{\Phi}_{l}(0)\right) e^{-R_{l j} z}\right. \\
& +\frac{\sigma^{2}}{2} D_{l j} \mathbf{L}_{l}{ }^{*}\left[\rho_{l 1}, \cdots, \rho_{l, m n}\right] \mathbf{\Phi}_{l}(0) e^{-R_{l j} z}-e^{-R_{l j} z} \star\left[\mathbf{Q}_{l j} \boldsymbol{\Gamma}_{l}(z)\right. \\
& \left.\left.-D_{l j} \sum_{i=1}^{m n} \mathbf{L}_{l}{ }^{*}\left[\rho_{l 1}, \cdots, \rho_{l i}\right](-1)^{m n-i}\left(\prod_{j=i}^{m n} T_{\rho_{l j}}\right) \boldsymbol{\Gamma}_{l}(z)\right]\right\}, \quad z \geq 0,
\end{aligned}
$$

where $\star$ represents the convolution operator. 
Since $\boldsymbol{\Phi}_{l}(z)=\mathbf{W}_{l}(u)=\mathbf{W}_{l}\left(z+d_{l-1}\right)$, we can obtain the following theorem from (5.27).

5.4. Theorem. If the claim-size distributions $F(x)$ and $G(x)$ both belong to the rational family, for $l=1,2, \cdots, L$, when $u \geq d_{l-1}$, the solutions of the equations (5.2) are given by

$$
\begin{aligned}
& \mathbf{W}_{l}(u)= \\
& \frac{1}{\left(\frac{\sigma^{2}}{2}\right)^{m n}} \sum_{j=1}^{m n\left(m_{1}+m_{2}+1\right)}\left\{\left(\frac{\sigma^{2}}{2} \mathbf{Q}_{l j} \mathbf{W}_{l}^{\prime}\left(d_{l-1}\right)+\mathbf{H}_{l j} \mathbf{W}_{l}\left(d_{l-1}\right)\right) e^{-R_{l j}\left(u-d_{l-1}\right)}\right. \\
& +\frac{\sigma^{2}}{2} D_{l j} \mathbf{L}_{l}{ }^{*}\left[\rho_{l 1}, \cdots, \rho_{l, m n}\right] \mathbf{W}_{l}\left(d_{l-1}\right) e^{-R_{l j}\left(u-d_{l-1}\right)} \\
& -e^{-R_{l j}\left(u-d_{l-1}\right)} \star\left[\mathbf{Q}_{l j} \boldsymbol{\Gamma}_{l}\left(u-d_{l-1}\right)\right. \\
& \left.\left.-D_{l j} \sum_{i=1}^{m n} \mathbf{L}_{l}^{*}\left[\rho_{l 1}, \cdots, \rho_{l i}\right](-1)^{m n-i}\left(\prod_{j=i}^{m n} T_{\rho_{l j}}\right) \boldsymbol{\Gamma}_{l}\left(u-d_{l-1}\right)\right]\right\}
\end{aligned}
$$

where $\mathbf{Q}_{l j}, D_{l j}$ and $\mathbf{H}_{l j}$ are given by (5.24), (5.25), respectively.

5.5. Remark. Let $l=1$ in (5.28), we have

$$
\mathbf{W}_{1}(u)=\frac{1}{\left(\frac{\sigma^{2}}{2}\right)^{m n-1}} \sum_{j=1}^{m n\left(m_{1}+m_{2}+1\right)} \mathbf{Q}_{1 j} \mathbf{W}_{1}^{\prime}(0) e^{-R_{1 j} u}, \quad u \geq 0 .
$$

Obviously, $\mathbf{W}(u)=\mathbf{W}_{1}(u)$ for $0 \leq u<d_{1}$. By virtue of $\mathbf{W}_{r}^{\prime}(u)=r \mathbf{W}_{r-1}(u)$. Thus, when $r=1, \mathbf{W}^{\prime}(u)=\mathbf{e}_{m n}$, that is, $\mathbf{W}^{\prime}\left(d_{l-1}\right)=\mathbf{e}_{m n}, l=2,3, \cdots, L$. So, differentiating (5.29) w.r.t. $u$ and letting $u=d_{1}$, we can determine $\mathbf{W}_{1}^{\prime}(0)$. Thus, $\mathbf{W}(u), 0 \leq u<d_{1}$ can be obtain.

\section{Numerical illustrations}

We now illustrate an application of the main conclusions in this paper with a numerical example. We suppose that the claim amounts from class 1 and class 2 have density functions, respectively,

$$
f(x)=\mu_{1} e^{-\mu_{1} x}, \quad \mu_{1}>0, x>0, \quad g(y)=\mu_{2} e^{-\mu_{2} y}, \quad \mu_{2}>0, y>0 .
$$

Hence, the Laplace transforms $\tilde{f}(s)=\frac{\mu_{1}}{s+\mu_{1}}, \tilde{g}(s)=\frac{\mu_{2}}{s+\mu_{2}}$. The inter-claim times from class 1 occur following a Poisson process with parameter $\lambda$, i.e. $\boldsymbol{\alpha}=(1), \mathbf{A}=(-\lambda), \mathbf{a}=$ $(\lambda)$, and inter-claim times from class 2 occur following a phase-type distribution with the following parameters: $\boldsymbol{\beta}=(1 / 2,1 / 2)^{\top}, \mathbf{B}=\operatorname{diag}\left(-\lambda_{1},-\lambda_{2}\right), \mathbf{b}=\left(\lambda_{1}, \lambda_{2}\right)^{\top}$. In addition, we assume that the multi-threshold layers $L=2$ with $0=d_{0}<d_{1}<d_{2}=\infty$. So, we have $\mathfrak{h}(s)=\left[\left(s+\mu_{1}\right)\left(s+\mu_{2}\right)\right]^{2}$ and $\mathbf{L}_{l}(s), l=1,2$ are given by

$$
\left(\begin{array}{cc}
\kappa(s)-\lambda_{1}+\frac{\lambda \mu_{1}}{s+\mu_{1}}+\frac{\lambda_{1} \mu_{2}}{2\left(s+\mu_{2}\right)} & \frac{\lambda_{1} \mu_{2}}{2\left(s+\mu_{2}\right)} \\
\frac{\lambda_{2} \mu_{2}}{2\left(s+\mu_{2}\right)} & \kappa(s)-\lambda_{2}+\frac{\lambda \mu_{1}}{s+\mu_{1}}+\frac{\lambda_{2} \mu_{2}}{2\left(s+\mu_{2}\right)}
\end{array}\right),
$$

where $\kappa(s)=\frac{\sigma^{2}}{2} s^{2}+c_{l} s-\delta-\lambda$. Let $\mu_{1}=1, \mu_{2}=2, \lambda=2, \lambda_{1}=1, \lambda_{2}=3, \sigma=1, \delta=$ $0.01, d_{1}=2, c_{1}=2$ and $c_{2}=1.5$. So, $\eta_{2}=0.5$, and the positive security loading conditions are satisfied. Under this hypothesis, the solutions of $\mathfrak{h}(s) \operatorname{det}\left[\mathbf{L}_{1}(s)\right]=0$ are $-R_{11}=$ $-6.0539,-R_{12}=-5.3665,-R_{13}=-2.0000,-R_{14}=-1.5891,-R_{15}=-0.5883,-R_{16}=$ $-0.0127, \rho_{11}=0.3244, \rho_{12}=1.2861$, and the solutions of $\mathfrak{h}(s) \operatorname{det}\left[\mathbf{L}_{2}(s)\right]=0$ are $-R_{21}=$ $-5.3915,-R_{22}=-4.6356,-R_{23}=-2.0000,-R_{24}=-1.5608,-R_{25}=-0.5600,-R_{26}=$ $-0.0079, \rho_{21}=0.6041, \rho_{22}=1.5517$. 
Differentiating (5.29) w.r.t. $u$, then letting $u=d_{1}$ and using $\mathbf{W}^{\prime}\left(d_{1}\right)=\mathbf{e}_{m n}$, we have $\mathbf{W}_{1}^{\prime}(0)=(90.7431,97.5835)^{\top}$. Substituting the value of $\mathbf{W}_{1}^{\prime}(0)$ into (5.29) and noting that the root $s=-2.0000$ is Singular, we have the expression of $\mathbf{W}(u)$ for $0 \leq u<2$,

$$
\begin{aligned}
& \mathbf{W}(u)= \\
& \left(\begin{array}{lllll}
-56.9599 & 48.0343 & -0.0078 & -6.5895 & 15.5229 \\
-85.1765 & 78.8988 & -1.2463 & -6.0170 & 13.5409
\end{array}\right)\left(\begin{array}{l}
e^{-6.0539 u} \\
e^{-5.3665 u} \\
e^{-1.5891 u} \\
e^{-0.5883 u} \\
e^{-0.0127 u}
\end{array}\right)
\end{aligned}
$$

Letting the initial conditions $\boldsymbol{\Xi}_{2}^{\prime}(0)=(1,0)^{\top}, \boldsymbol{\Xi}_{2}(0)=(0,1)^{\top}$ and $\boldsymbol{\Xi}_{2}^{\prime}(0)=(0,1)^{\top}$, $\boldsymbol{\Xi}_{2}(0)=(1,0)^{\top}$, respectively, by virtue of the asymptotic behaviour of $\mathbf{W}(u), u \geq 2$, we get the following two linearly independent solutions from (5.21) when $u \geq 2$,

$$
\begin{aligned}
& \Theta_{21}(u)=\left(\begin{array}{ccccc}
6.5218 & -2.9357 & -0.0069 & -0.0053 & -1.4173 \\
59.4285 & 1.1493 & -0.0164 & 0.0118 & -1.4228
\end{array}\right)\left(\begin{array}{l}
e^{-5.3915(u-2)} \\
e^{-4.6356(u-2)} \\
e^{-1.5608(u-2)} \\
e^{-0.5600(u-2)} \\
e^{-0.0079(u-2)}
\end{array}\right) \\
& \boldsymbol{\Theta}_{22}(u)=\left(\begin{array}{ccccc}
2.1362 & 21.4218 & -0.0057 & -0.0046 & -3.4694 \\
19.4654 & -8.3868 & -0.0135 & 0.0103 & -3.4830
\end{array}\right)\left(\begin{array}{l}
e^{-5.3915(u-2)} \\
e^{-4.6356(u-2)} \\
e^{-1.5608(u-2)} \\
e^{-0.5600(u-2)} \\
e^{-0.0079(u-2)}
\end{array}\right)
\end{aligned}
$$

Combining (5.5) with (6.1) and utilizing the boundary condition $\left.\mathbf{W}(u)\right|_{u=d_{1}^{-}}=\left.\mathbf{W}(u)\right|_{u=d_{1}^{+}}$, then solving the linear equations, we have $k_{21}=-0.4243, k_{22}=$ -1.7921 . Thus, we obtain $\mathbf{W}(u), u \geq 2$,

$$
\begin{aligned}
& \mathbf{W}(u)=\left(\begin{array}{l}
50 \\
50
\end{array}\right)+ \\
& \left(\begin{array}{ccccc}
-6.5956 & -37.1448 & 0.0131 & 0.0105 & 6.8190 \\
-60.1005 & 14.5425 & 0.0312 & -0.0235 & 6.8457
\end{array}\right)\left(\begin{array}{l}
e^{-5.3915(u-2)} \\
e^{-4.6356(u-2)} \\
e^{-1.5608(u-2)} \\
e^{-0.5600(u-2)} \\
e^{-0.0079(u-2)}
\end{array}\right)
\end{aligned}
$$

Last, since $\boldsymbol{\gamma}=\boldsymbol{\beta} \otimes \boldsymbol{\alpha}=(1 / 2,1 / 2)^{\top}$, we can obtain $W(u)$ by $W(u)=\boldsymbol{\gamma}^{\top} \mathbf{W}(u)$, viz,

$$
W(u)=\left\{\begin{array}{c}
-71.0682 e^{-6.0539 u}+63.4665 e^{-5.3665 u}-0.6271 e^{-1.5891 u} \\
-6.3033 e^{-0.5883 u}+14.5319 e^{-0.0127 u}, \quad 0 \leq u<2, \\
50-33.3481 e^{-5.3915(u-2)}-11.3011 e^{-4.6356(u-2)} \\
+0.0222 e^{-1.5608(u-2)}-0.0065 e^{-0.5600(u-2)} \\
+6.8323 e^{-0.0079(u-2)}, \quad u \geq 2 .
\end{array}\right.
$$

\section{Acknowledgements}

This work was supported by the National social Science Foundation of China (17BJY206). 


\section{References}

[1] Albrecher, H. Claramunt, M.M. and Mármol, M. On the distribution of dividend payments in a Sparre Andersen model with generalized Erlang(n) interclaim times, Insurance: Mathematics and Economics 37, 324-334, 2005.

[2] Asmussen, S. and Albrecher, H. Ruin probabilities, second ed. World Scientific, New Jersey. 2010.

[3] Chueng, E.C.K. and Landriault, D. Perturbed MAP risk models with dividend barrier strategies, Journal of Applied Probability 46, 521-541, 2009.

[4] Dickson, D.C.M. and Hipp, C. On the of ruin for Erlang(2) risk processes, Insurance: Mathematics and Economics 29, 333-344, 2001.

[5] Gao, H. and Yin, C. The perturbed Sparre Andersen model with a threshold dividend strategy, Journal of Computational and Applied Mathematics 220, 394-408, 2008.

[6] Gerber, H.U. and Shiu, E.S.W. 2005. The time value of ruin in a Sparre Andersen model, North American Actuarial Journal 9, 49-69, 2005.

[7] Ji, L. and Zhang, C. The Gerber-Shiu penalty functions for two classes of renewal risk processes, Journal of Computational and Applied Mathematics 233, 2575-2589, 2010.

[8] Jiang, W.Y. and Yang, Z.J. The phase-type risk model perturbed by diffusion under a threshold dividend strategy, Acta Mathematicae Applicatae Sinica English Series 29, 215-224, 2013.

[9] Jiang, W.Y. and Yang, Z.J. The expected discounted penalty function for two classes of risk processes perturbed by diffusion with multiple thresholds, Indian Journal of Pure and Applied Mathematics 45, 479-295, 2014.

[10] Li, S. The distribution of the dividend payments in the compound Poisson risk model perturbed by diffusion, Scandinavian Actuarial Journal 26, 73-85, 2006.

[11] $\mathrm{Li}, \mathrm{S}$. and $\mathrm{Lu}, \mathrm{Y}$. On the expected discounted penalty functions for two classes of risk processes, Insurance: Mathematics and Economics 36, 179-193, 2005.

[12] Lin, X.S. and Sendova, K.P. The compound Poisson risk model with multiple thresholds, Insurance: Mathematics and Economics 42, 617-627, 2008.

[13] $\mathrm{Lu}, \mathrm{Y}$. and $\mathrm{Li}, \mathrm{S}$. The Markovian regime-switching risk model with a threshold divident strategy, Insurance: Mathematics and Economics 44, 296-303, 2009.

[14] Yang, H. and Zhang, Z.M. Gerber-Shiu discounted penalty function in a Sparre Andersen model with multi-layer dividend strategy, Insurance: Mathematics and Economics 42, 984991, 2008.

[15] Zhang, Z.M. Li, S. and Yang, H. The Gerber-Shiu discounted penalty functions for a risk model with two classes of claims, Journal of Computational and Applied Mathematics 230, 643-655, 2009.

[16] Wan, N. Dividend payments with a threshold dividend strategy in the compound Poisson risk model perturbed by diffusion, Insurance: Mathematics and Economics 40, 509-532, 2007. 